\title{
Importância da consulta de Enfermagem para orientações de preparo para a realização de colonoscopia
}

\author{
Importance of the Nursing consultation for orientation on how to prepare for colonoscopy \\ Importancia de la consulta de Enfermería para las orientaciones de preparo para realización de \\ colonoscopia
}

Débora Machado Nascimento do Espírito Santo ORCID: https://orcid.org/0000-0003-0533-0335 Hospital de Clínicas de Porto Alegre, Brasil E-mail:dsanto@hcpa.edu.br

Lisiane Paula Sordi Matzenbacher ORCID: https://orcid.org/0000-0002-1407-7717 Hospital de Clínicas de Porto Alegre, Brasil E-mail:1sordi@hcpa.edu.br

Rosaura Soares Paczek ORCID: https://orcid.org/0000-0002-4397-1814 Hospital de Clínicas de Porto Alegre, Brasil E-mail:rspaczek@gmail.com

Carina Galvan

ORCID: https://orcid.org/0000-0003-2111-5432 Hospital de Clínicas de Porto Alegre, Brasil E-mail:cgavan@hcpa.edu.br

Ana Karina Silva da Rocha Tanaka

ORCID: https://orcid.org/0000-0003-2488-3656 Universidade Federal do Rio Grande do Sul, Brasil

E-mail:anakarinatanaka@gmail.com

Ana Maria Pagliarini

ORCID: https://orcid.org/0000-0003-4585-4846 Universidade Federal do Rio Grande do Sul, Brasil E-mail:apagliarini@hcpa.edu.br

\begin{abstract}
Resumo
Objetivo: relatar a importância da consulta de Enfermagem para orientações de preparo para a realização de colonoscopia. Método: estudo descritivo, do tipo relato de experiência, sobre a atuação e as vivências das enfermeiras atuantes na unidade de endoscopia, em um hospital de grande porte localizado no Sul do Brasil, realizado em maio de 2021. Resultados: a colonoscopia permite a visualização completa da mucosa do cólon e íleo terminal e é considerada um método completo na investigação das doenças colorretais. O preparo intestinal adequado é um fator determinante para a capacidade de realização do exame e a duração do procedimento. $O$ enfermeiro exerce papel fundamental na assistência prestada ao indivíduo nos dias que antecedem o exame relacionado às orientações e condutas para o seu êxito. Estas devem ser individuais e específicas para cada paciente, considerando a faixa etária, a comorbidade, o grau de instrução e o grau de dificuldade para a realização do preparo. Considerações finais: baseado na prática realizada pelas enfermeiras, observou-se que a falha nas orientações ou o não cumprimento adequado do preparo por parte dos pacientes pode ocasionar algumas complicações durante o exame ou até inviabilizar a realização do mesmo e, para minimizar os possíveis contratempos que possam ocorrer, a atuação de uma enfermeira treinada e habilitada garante a segurança do paciente e proporciona uma assistência integral, de forma planejada e adequada, para a realização do exame.
\end{abstract}

Palavras-chave: Enfermagem; Educação em saúde; Colonoscopia; Consulta de enfermagem.

\begin{abstract}
Objective: to report the importance of the Nursing consultation for guidance in preparation for colonoscopy. Method: a descriptive study, of the experience report type, about the performance and experiences of nurses working in the endoscopy unit, in a large hospital located in the South of Brazil, carried out in May 2021. Results: colonoscopy allows complete visualization of the mucosa of the colon and terminal ileum and is considered a complete method in the investigation of colorectal diseases. The adequate intestinal preparation is a determining factor for the ability to perform the exam and the duration of the procedure. The nurse plays a fundamental role in the assistance provided to
\end{abstract}


the individual in the days preceding the exam related to the guidelines and conducts for its success. These should be individual and specific for each patient, considering the age group, comorbidity, level of education, and the degree of difficulty to perform the preparation. Final considerations: based on the practice carried out by the nurses, it was observed that the lack of guidance or the patients' failure to adequately prepare for the exam can cause some complications during the exam or even make it unfeasible, and to minimize possible setbacks that may occur, the performance of a trained and qualified nurse ensures patient safety and provides comprehensive care, in a planned and appropriate way, for the exam.

Keywords: Nursing; Health education; Colonoscopy; Office nursing.

\section{Resumen}

Objetivo: relatar la importancia de la consulta de Enfermería para orientaciones de preparo para la realización de colonoscopia. Método: estudio descriptivo, tipo relato de experiencia, sobre el desempeño y vivencias de enfermeras que laboran en la unidad de endoscopia, en un gran hospital ubicado en el sur de Brasil, realizado en mayo de 2021. Resultados: la colonoscopia permite la visualización completa de la mucosa colónica y íleo terminal y se considera un método completo en la investigación de enfermedades colorrectales. El preparo intestinal adecuado es un factor determinante para la capacidad de realización del examen y la duración del procedimiento. El enfermero ejerce un papel fundamental en la asistencia brindada al individuo en los días previos al examen, relacionada con las pautas y conductas para su éxito. Estos deben ser individuales y específicos para cada paciente, considerando el grupo de edad, la comorbilidad, el nivel de estudios y el grado de dificultad para realización del preparo. Consideraciones finales: con base en la práctica realizada por las enfermeras, se observó que la falla en las orientaciones o en el no cumplimiento adecuado en el preparo por parte de los pacientes puede ocasionar algunas complicaciones durante el examen o incluso hacerlo inviable y, para minimizar posibles retrocesos que puedan ocurrir, la actuación de una enfermera capacitada y calificada garantiza la seguridad del paciente y brinda una atención integral, de manera planificada y adecuada, para la realización del exámen.

Palabras clave: Enfermería; Educación en salud; Colonoscopía; Consulta de enfermería.

\section{Introdução}

O uso da colonoscopia para o rastreamento e a prevenção do câncer colorretal é considerado o padrão-ouro mundial para a investigação desses casos. No Brasil, o câncer colorretal é apontado como o terceiro tipo de câncer mais constante entre homens e o segundo entre mulheres. É considerado também como a maior causa de morte por câncer no mundo (Lima et al., 2021).

A colonoscopia permite a visualização completa da mucosa do cólon e íleo terminal, sendo considerada um método completo na investigação e no tratamento de doenças colorretais, possui custo elevado, depende de sedação e de um bom preparo intestinal (Marcacini et al., 2015). O preparo intestinal adequado é um fator determinante para a capacidade de realização do exame, assim como a duração do procedimento (Andrade et al., 2020). Isso auxilia também o diagnóstico e as abordagens terapêuticas necessárias, pois possibilita a realização do exame de forma segura, com a introdução do aparelho sob visão direta, evitando que lesões passem despercebidas (Andrade et al., 2017; Guinhazi et al., 2019; Sánchez-del-Río et al.,2018).

Alguns fatores têm colaborado para a qualidade do exame, como a técnica adequada, a evolução dos aparelhos com imagens de qualidade e profissionais com habilidades práticas para a realização da colonoscopia (Kaiser-Junior et al., 2018).

O preparo adequado tornou-se a parte mais sensível da colonoscopia, pois a sua visualização depende exclusivamente desse cuidado. Além de facilitar a identificação de lesões na mucosa, reduz a possibilidade de repetição do exame por presença de resíduos. A orientação clara e precisa sobre o preparo irá influenciar a aceitação do paciente a seguir a dieta e o uso dos medicamentos, auxiliando para um exame de qualidade e diminuindo as complicações (Diniz, 2019).

Tendo como objetivo a limpeza completa da luz intestinal para uma melhor visualização e inspeção da mucosa em toda a sua extensão. Para isso, um protocolo de preparo definindo o tipo de dieta, a dosagem e o horário dos laxantes é essencial. Esse preparo deve iniciar-se 12 a 72 horas antes do exame, com algumas observações, como: dieta líquida sem resíduos, restrição de fibras, frutas e legumes, sendo permitidos líquidos claros e o uso de laxativos para garantir a eficácia 
(Milke \& Welfe, 2019).

Alguns pacientes necessitam de orientações especiais da equipe para a realização da colonoscopia, como no caso de pacientes com desfibriladores e marcapassos implantados, que, durante o exame, podem ter o seu funcionamento comprometido (Paczek et al., 2020; Inca, 2020).

A consulta de Enfermagem é uma combinação entre os conhecimentos humano, científico e empírico com o intuito de prestar uma assistência de melhor qualidade e que se efetiva no cuidado ao indivíduo, à família e à comunidade. Compreende também os processos e os métodos envolvidos nos cuidados de Enfermagem. É a dinâmica das ações sistematizadas e interrelacionadas com o intuito de assistir o ser humano (Dantas, 2016).

O enfermeiro exerce papel fundamental na assistência prestada ao indivíduo, nos dias que antecedem o exame, relacionado às orientações e condutas para o seu êxito. Estas devem ser individuais e específicas para cada paciente, considerando a faixa etária, a comorbidade, o grau de instrução e o grau de dificuldade para a realização do preparo (Andrade et al., 2017).

Um preparo inadequado impacta negativamente os custos e riscos aos quais o paciente é submetido. Além disso, limita também a sensibilidade para a identificação de pólipos e lesões, os quais podem deixar de ser diagnosticados precocemente (Amorin et al., 2020).

O objetivo deste estudo é relatar a atuação das enfermeiras na consulta de Enfermagem para orientações sobre o preparo da colonoscopia.

\section{Metodologia}

Trata-se de um estudo descritivo, do tipo relato de experiência, sobre a atuação e as vivências das enfermeiras na unidade de endoscopia, vivenciado em maio de 2021, realizado durante a consulta de Enfermagem para a orientação de preparo da colonoscopia. O local do estudo foi o centro cirúrgico ambulatorial de um hospital de grande porte localizado na região Sul do Brasil, o qual possui cinco salas de procedimentos endoscópicos e oito salas cirúrgicas, 15 leitos de recuperação pós-anestésica adulto e cinco pediátricos. Conta também com oito leitos para a recuperação de pacientes pós-sedação de procedimentos endoscópicos.

O relato de experiência reúne diversos elementos descritos, por meio de uma experiência vivida, capazes de englobar processos e produções, aplicando a prática baseada em evidência. A produção científica está permanentemente desafiada a desenvolver-se por meio da pesquisa, das metodologias e das relações multidisciplinares (Minayo, 2012).

\section{Relato da Experiência}

No local de estudo, as colonoscopias são realizadas em nível ambulatorial e também em pacientes internados. São realizados em torno de 60 exames ao mês e, para os pacientes que realizam o procedimento em nível ambulatorial, é realizada uma consulta de Enfermagem na qual o paciente recebe informações com a finalidade de receber orientações sobre o preparo necessário.

A marcação da consulta de Enfermagem é realizada na secretaria do centro cirúrgico ambulatorial após a consulta ambulatorial com o médico assistente, o gastroenterologista ou o proctologista. É entregue, ao paciente, um formulário contendo a data e o horário da realização do exame, assim como a data da consulta de Enfermagem para receber as orientações do preparo da colonoscopia. Essas consultas são realizadas três vezes na semana: segundas, quartas e quintas-feiras, com antecedência de até uma semana do exame, tempo suficiente para iniciar o preparo adequado.

A enfermeira tem acesso aos agendamentos pelo sistema informatizado da instituição no qual constam o nome 
completo e o prontuário dos pacientes. As consultas são realizadas conforme a ordem de chegada dos pacientes, em uma sala própria, individualizada e específica para este atendimento.

As instruções realizadas durante a consulta de Enfermagem referem-se ao cumprimento rigoroso da dieta e é fornecido um folheto no qual constam todas as informações. No primeiro dia de preparo, o paciente é orientado a ingerir líquidos, como chá, chimarrão, água, café preto, refrigerantes, água de coco e bebidas isotônicas de cor clara à vontade, e livre demanda dos seguintes sólidos: ovos cozidos; rosca de polvilho; gelatina de coloração clara; merengue; macarrão instantâneo sem o tempero e peito de frango pequeno cozido com água e sal. É reforçada a importância da ingesta hídrica durante os dias em que estiver realizando a dieta. Deve-se fazer uso de um comprimido laxativo, às $8 \mathrm{~h}$ e às $20 \mathrm{~h}$, ainda no primeiro dia do preparo. No segundo dia, mantém-se a necessidade de seguir a mesma dieta do primeiro dia.

O paciente recebe os frascos de manitol 10\%, sendo orientado de como utilizar e preparar a solução a ser ingerida. Orienta-se a utilizar o suco coado de dois limões e observa-se que, para a limpeza intestinal ser efetiva, é necessário ingerir 750 $\mathrm{ml}$ da solução de seis a oito horas antes do procedimento. Orienta-se a realizar a mistura com limão devido ao gosto adocicado do manitol, que, muitas vezes, provoca náuseas e vômitos. Esta solução deve ser ingerida dentro de um período de uma hora após o seu preparo para que obtenha o efeito laxativo e atinja o objetivo da limpeza intestinal adequada.

A solução de manitol não deve ser acondicionada em refrigerador. Deve-se mantê-la sempre em temperatura ambiente, principalmente, no inverno rigoroso da região Sul do Brasil, pois esta solução pode cristalizar e, quando isso acontece, os pacientes são instruídos a desfazer os cristais, realizando a imersão dos frascos em banho-maria, para que ocorra a dissolução antes da ingestão.

O paciente é orientado que, após o consumo da solução de manitol, irão ocorrer vários episódios de diarreia, podendo levá-lo ao banheiro de dez a 15 vezes. Este é o efeito desejado para que o intestino fique limpo. As últimas evacuações devem ser somente líquidas, de coloração amarelada, sem a presença de fezes.

Durante a consulta de Enfermagem, além de o paciente receber todas as orientações referentes à dieta e ao uso de laxativos, a enfermeira também realiza uma anamnese, coletando dados importantes sobre as comorbidades, o uso de medicamentos e os hábitos de vida. Alguns medicamentos específicos, como os antiagregantes plaquetários e os diuréticos, por vezes, necessitam ser suspensos. Nestes casos, o paciente é orientado a agendar uma consulta ou informar o médico assistente.

É entregue, ao paciente, o folheto com a data do procedimento, a data do início da dieta, os horários dos laxativos e a relação dos alimentos liberados para o consumo. O mesmo é informado que, no dia do exame, deverá comparecer com duas horas de antecedência ao horário agendado no centro cirúrgico ambulatorial, com um acompanhante maior de 18 anos, e que receberá sedação endovenosa para a realização do procedimento, as informações de como o procedimento é realizado e que, após recuperar-se da sedação, terá alta para o seu domicílio. Nesse formulário, consta também um número de telefone de contato do centro cirúrgico, que funciona durante as 24 horas do dia, para sanar dúvidas pendentes em relação ao preparo da colonoscopia.

Importante ressaltar que o preparo deve ser seguido de forma rigorosa pois, caso não seja realizado adequadamente, o intestino poderá apresentar resíduos fecais, o que impossibilita a visualização de sua parede, podendo o exame não ser realizado ou ser inspecionado de forma parcial. Isso dará ensejo a um reagendamento, tendo o paciente que realizar todo o preparo novamente.

\section{Discussão}

As informações dadas ao paciente devem ser seguidas rigorosamente no que se refere à necessidade de manter corretamente a dieta específica, que inclui líquidos e alimentos exclusivos de fácil digestão, todos descritos no formulário. 
Estas orientações são prestadas pela enfermeira, que deve utilizar uma linguagem de fácil compreensão para atender à realidade e à diversidade dos pacientes atendidos no Sistema Único de Saúde (SUS).

O paciente bem orientado tem menos dificuldades para seguir as instruções e maior probabilidade de sucesso no procedimento. Uma orientação adequada da Enfermagem no preparo para a colonoscopia auxilia a limpeza intestinal e possibilita um melhor diagnóstico (Andrade et al., 2017).

Uma dieta com baixo teor de fibras deve ser iniciada dois dias antes da colonoscopia. O preparo intestinal adequado está relacionado com o sucesso desse procedimento, facilitando a visualização da parede intestinal, reduzindo o tempo do exame e, consequentemente, o risco de complicações (Zhang et al., 2018). Com isso, enfatiza-se a importância da educação das equipes de saúde para auxiliar e padronizar as condutas frente à preparação intestinal para os exames de colonoscopia (Yang et al., 2014).

As complicações mais frequentes são a perfuração e a hemorragia com maior índice em pacientes com graves comorbidades. Podem ser decorrentes do preparo intestinal, da lesão do mesentério, de complicações cardiovasculares e infecções. As mais leves são: queixas de dor abdominal, flatulência, náuseas e sangramento intestinal sem repercussão hemodinâmica (Coser et al., 2018). Também são identificadas complicações durante os dias que antecedem o exame, devido à realização do preparo, que são atribuídas ao jejum, ao distúrbio eletrolítico, à dor abdominal, às náuseas e aos vômitos (Gaspar \& Morais, 2020).

A capacitação individual dos pacientes, realizada por enfermeiros, possibilita melhores resultados da educação e favorece o suporte emocional. O risco de orientações realizadas apenas por meio de vídeos impossibilita a interação dos pacientes, tornando, por vezes, as informações incompressíveis (Veldhuijzen et al., 2017).

Fatores como a alfabetização, as comorbidades e a baixa renda familiar foram relacionados com o mau preparo intestinal. Outro fator apontou que a compreensão de como acontece o exame de colonoscopia pode exercer influência significativa na qualidade do preparo (Liu et al., 2018). Com a identificação dessas causas, torna-se necessário implementar medidas educativas e estratégias para reduzir o risco de preparo inadequado (Antonio-Cisneros et al., 2016).

Por fim, ressalta-se que o enfermeiro, partindo de uma avaliação individualizada, deverá reconhecer as dificuldades de compreensão de cada paciente e elaborar estratégias para auxiliar o entendimento, e o uso de folhetos explicativos têm auxiliado para sanar dúvidas posteriores à consulta (Diniz et al.,2019).

\section{Considerações Finais}

Baseada na prática realizada pelas enfermeiras, observou-se que a falha nas orientações ou o não cumprimento adequado do preparo por parte do paciente pode ocasionar algumas complicações durante o exame ou até inviabilizar a realização do mesmo. Com isso, faz-se necessária uma equipe de enfermeiros capacitados, proporcionando uma assistência integral.

A orientação adequada sobre o preparo de colonoscopia durante a consulta de Enfermagem proporciona um diagnóstico eficaz ao paciente. A adesão efetiva garante o sucesso do exame e possibilita um tratamento precoce, garantindo a segurança e a qualidade assistencial.

\section{Referências}

Amorim, T., Gomes, L., Coelho, L., Paiva, A., Salimena, A. M., Cassimiro, B., et al.(2020). Ações de Enfermagem que contribuem para o preparo da colonoscopia: revisão integrativa. Revista Enfermagem Atual In Derme, 94(32), e-020062. https://doi.org/10.31011/reaid-2020-v.94-n.32-art.914. 
Andrade, L. C., Baldin, J. A., Cardoso, M.N., Onibene, B. C. A., Azevedo, A. \& Andrade, B. B. P. (2020). Explosão de cólon durante colonoscopia. Braz. J. Hea. Rev., 3 (5), 12200-12205.

Antonio-Cisneros, A., Antonio-Manrique, M., Chávez-García, M. Á., Hernández-Velázquez, N. N., Pérez-Valle, E., Pérez-Corona, T., et al.(2016). Factores pronósticos de preparación intestinal inadecuada en colonoscopia. Rev Hosp Jua Mex. ,83(3):75-79.

Coser, R. B., Dalio, M. B., Martins, L. C. P., Alvarenga, G. F., Cruz, C. A., Imperiale, A. R. et al. (2018). Complicações em colonoscopia: experiência uniinstitucional com 8968 pacientes. Rev. Col. Bras. Cir., 45(4). https://doi.org/10.1590/0100-6991e-20181858.

Dantas, C. N., Santos, V. E. P. T. \& Francis, S. V. A. (2016). Consulta de enfermagem como tecnologia do cuidado à luz dos pensamentos de Bacon e Galimberti. Texto \& Contexto - Enfermagem, 25(1). https://doi.org/10.1590/0104-0707201500002800014.

Diniz, T. S. R.(2019). Construção de folheto educativo e orientação via telefone para o preparo da colonoscopia: estudo clínico, controlado e randomizado. Dissertação (Mestrado em Enfermagem). Universidade Estadual Paulista Júlio de Mesquita Filho, Botucatu, São Paulo.

Gaspar, H., \& Morais, V. (2020). Colonoscopia: quando a preparação se torna uma complicação. Revista Portuguesa de Medicina Geral e Familiar, 36(2), 186-93. http://dx.doi.org/10.32385/rpmgf.v36i2.12461.

Guinhazi, N. P., Silva, R. A., Tavares, L. F., Brito, A. P. O., \& Brito, M. V. H.(2019). Indicações e condutas de rastreamento de pólipos intestinais: uma revisão de literatura. Revista Artigos.Com, e158.

INCA (2020). ABC do Câncer: abordagens básicas para o controle do câncer / Instituto Nacional de câncer José Alencar Gomes da Silva; organização Mario Jorge Sobreira da Silva. (5a ed.), https://www.inca.gov.br/sites/ufu.sti.inca.loal/files//media/document//livro-abc-6-edicao-2020.pdf

Kaiser-Júnior, R. L., Quadros, L. G., Flamini-Júnior, M., Faria, M. A. G., Campo, J. C. O., Oliveira, V. L. et al. (2018). Nova técnica de preparo intestinal para colonoscopia: estudo clínico comparativo entre Aquanet e manitol. ABCD. Arquivos Brasileiros de Cirurgia Digestiva, $31(3)$, e1393. DOI: https://doi.org/10.1590/0102-672020180001e1393.

Lima, J. A., Miserani, M. P. C. L. C. \& Coelho, J. C. C. G. P. (2021). Frequência de adenomas serrilhados em pacientes submetidos a colonoscopia em uma unidade ambulatorial de alta demanda em Belo Horizonte. MG. Open Journal Systems, 5(1).

Liu, C., Song, X., \& Hao, H. (2018). Educational Video Followed by Retelling Bowel Preparation Process to Improve Colonoscopy Bowel Preparation Quality: A Prospective Nursing Intervention Study. Med Sci Monit, 24, 6029-6037. 10.12659/MSM.909572..

Marcacini, S. F. B., Barichello, E. \& Barbosa, M. H.(2015). Perfil dos pacientes submetidos à colonoscopia em um hospital universitário. Enfermería Global,14 (39), 62-71.

Milke, P. G.\& Welfe, M. (2019). Videocolonoscopia: os cuidados na visão da equipe de enfermagem. Revista Inova Saúde, 9(1).

Minayo, M. C. S. (2012). Análise qualitativa: teoria, passos e fidedignidade. Ciência \& Saúde Coletiva. 17(3), 621-626.

DOI: https://doi.org/10.1590/S1413-81232012000300007.

Paczek, R. S., Brum, B. N., Galvan, C., Espírito Santo, D. M. N., Brito, D. T., Prates, E. I. S., et al. (2020). Cartilha de orientação para preparo de colonoscopia. UFRGS.

Sánchez-del-Río, A., Pérez-Romero, S., López-Picazo, J., Alberca-de-las-Parras, F., Júdez, J. \& León-Molina, J. (2018). Indicadores de calidad en colonoscopia. Procedimiento de la colonoscopia. Revista Española de Enfermedades Digestivas, 110(5), 316-326.

DOI: http://dx.doi.org/10.17235/reed.2018.5408/2017

Veldhuijzen, G., Klemt-Kropp, M., Noomen, C., Van Esch, A. A., Tjwa, E. T., \& Drenth, J. (2017). Computer-assisted instruction before colonoscopy is as effective as nurse counselling, a clinical pilot trial. Endoscopy international open, 5(8), E792-E797.10.1055/s-0043-110813.

Yang, D., Summerlee, R., Rajca, B.,Williamson, J.B., Jennifer LeLaurin, J. \& Lasheaka McClellan, L. et al. (2015). A pilot study to evaluate the feasibility of implementing a split-dose bowel preparation for inpatient colonoscopy: a single-center experience. BMJ Open Gastroenterology,1(1):e00006. http://dx.doi.org/10.1136/bmjgast-2014-000006.

Zhang, Y. Y., Niu, M., Wu, Z. Y., Wang, X. Y., Zhao, Y. Y., \& Gu, J. (2018). The incidence of and risk factors for inadequate bowel preparation in elderly patients: A prospective observational study. Saudi journal of gastroenterology : official journal of the Saudi Gastroenterology Association, 24(2), 87-92. 10.4103/sjg.SJG_426_17. 